\title{
Microsurgical treatment of parafalcine meningiomas: a retrospective study of 126 cases
}

This article was published in the following Dove Press journal:

OncoTargets and Therapy

\author{
Xiangyi Kong ${ }^{1,2, *}$ \\ Shun Gong ${ }^{3-5, *}$ \\ I-Ting Lee C.7,* $^{6}$ \\ Yi Yang'
}

'Department of Neurosurgery, Peking Union Medical College Hospital, Chinese Academy of Medical Sciences, Beijing, People's Republic of China; ${ }^{2}$ Department of Breast Surgical Oncology, National Cancer Center/ National Clinical Research Center for Cancer/Cancer Hospital, Chinese Academy of Medical Sciences and Peking Union Medical College, Beijing, People's Republic of China; ${ }^{3}$ Department of Neurosurgery, The General Hospital of Shenyang Military, Army Institute of Neurology, Shenyang, People's Republic of China; ${ }^{4}$ Department of Neurosurgery, Shanghai Institute of Neurosurgery, PLA Institute of Neurosurgery, Shanghai Changzheng Hospital, Second Military Medical University, Shanghai, People's Republic of China; ${ }^{5}$ Department of Radiology, Brigham and Women's Hospital, Harvard Medical School, Boston, MA, USA; ${ }^{6}$ Peking University Health Science Center, Peking University, Beijing, People's Republic of China; ${ }^{7}$ Department of Dermatology, Beijing Hospital of the Ministry of Health, Beijing, People's Republic of China

*These authors contribute equally to this work

Correspondence: Yi Yang Department of Neurosurgery, Peking Union Medical College Hospital, Chinese Academy of Medical Sciences, I Shuaifuyuan, Dongcheng District, Beijing 100730, People's Republic of China

Tel +861069152530

Email yangyipumc@163.com
Objective: To discuss the diagnosis, operation methods, and clinical effects of parafalcine meningiomas.

Methods: The clinical and preoperative imaging characteristics, operative methods, and effects of operations of 126 cases of parafalcine meningiomas were respectively discussed.

Results: G1 resection was achieved in 13 cases, G2 in 105 cases, G3 in four cases, and G4 in four cases, with no deaths. Among these, there were 16 patients with dyskinesia of the contralateral extremities after surgery, but they recovered after several months.

Conclusion: In order to avoid postoperative complications, we consider it vital to analyze the patients' condition, the anatomy of venous drainage in by digital subtractional angiography, the relationship between tumor location and brain tissue according to MRI, and to remove the tumor in an adequately exposed surgical field.

Keywords: parafalcine meningioma, image-based types, microsurgery

\section{Introduction}

Parafalcine meningioma is a common meningioma located in the cerebral longitudinal fissure, originating from the cerebral falx, with the third highest morbidity among all the meningiomas, accounting for approximately $11 \%-14 \%$ deaths, ranking only second to the cerebral convexity meningioma and parasagittal meningioma (the term parasagittal meningioma applies to those tumors involving the sagittal sinus and the adjacent convexity dura and falx, only the lateral wall of the sinus may be involved or the tumor can may grow to partially or completely occlude the sinus). ${ }^{1}$ Parafalcine meningiomas are usually buried deep in the cerebral parenchyma, which does not permit an adequately exposed surgical field, this coupled with the influence of the position of the central sulcus vein lead to great difficulty in terms of surgery. ${ }^{2}$ A total of 126 patients with parasagittal meningioma were admitted to our hospital from January 2010 to June 2016, all of them were treated with resection along the longitudinal fissure by means of microsurgery, as reported in the following sections.

\section{Methods}

\section{Ethical approval}

Because this study was a retrospective study and did not involve any experimental interventions, according to the rules of the ethics committee of Peking Union Medical College Hospital, it did not require special ethics approval. All the patients signed written informed consent forms for their medical records to be reviewed for our study. 


\section{Clinical data}

\section{General data}

Of the 126 cases, 46 were male and 80 were female; age ranged from 31 to 83 years old with an average age of 50.1 years. The course of disease lasted from 10 days to 6 years, averaging 1.8 years.

\section{Symptoms and vital signs}

Among the 126 cases, there were 71 cases whose initial symptom was headache, 17 cases whose initial symptom was epileptic seizure, 25 cases whose initial symptom was decreased muscle force of contralateral limb, and 13 cases whose initial symptom was paresthesia. It was revealed by physical examination that there were 29 cases with visual dysfunction, 17 cases with positive pyramidal signs, and eight cases with positive result of ataxia.

\section{Imaging data}

Among the 126 cases, there were 101 cases who received a preoperative CT plain scan of the skull which revealed that there were 55 cases that had a uniform density of parafalcine space-occupying parafalcine tumors, 29 cases that had a relatively high density tumor and 17 cases that had a non-uniform density tumor, and there were 13 cases that peritumoral edema complication; all of the 101 cases were shown, by the enhanced scan, to have enhancement of varying degrees. There were 13 cases whose maximum diameter of tumor was more than $4 \mathrm{~cm}, 80$ cases ranging from 2 to $4 \mathrm{~cm}$, and 33 cases less than $2 \mathrm{~cm}$, averaging $2.6 \mathrm{~cm}$ in maximum diameter.

All of the 126 cases received an MRI contrast enhanced scan, revealing a quasi-circular or lobulated parafalcine tumor with a clear border; 71 cases were found to have a slightly low signal and 55 cases were found to have an equal signal by tumor T1 weighted image; 92 cases were found to have a high signal and 34 cases were found to have an equal signal by tumor $\mathrm{T} 2$ weighted image, which showed a significant enhancement after contrast enhancement.

Sixty-seven cases had an edema zone with a high T2 signal around the tumor, among them, there were 21 cases with widespread edema; 92 cases were found to have the dural tail sign. According to the MRI findings (sagittal view), the cerebral falx was divided into three parts by the lambdoidal suture and coronal suture, 42 cases' tumors were entirely located at the anterior third of cerebral falx, 122 at the middle third of cerebral falx, and 17 at the posterior third of cerebral falx; there were 25 cases whose tumors were located over both anterior and middle thirds of cerebral falx, and there were eight cases whose tumors were located over both middle and posterior thirds of cerebral falx; there were four cases with multiple meningiomas, of which the bigger one was located at the posterior third of cerebral falx and the smaller one was located at the anterior third of cerebral falx.

According to MRI findings (coronal view), the parafalcine meningiomas were divided into three types: type-I, a tumor at one side of cerebral falx without compression and displacement of cerebral falx, 71 cases had this type; type-II, a tumor at one side of cerebral falx, part of which evidently protruded to the opposite side, 34 cases had this type; type-III, a tumor which grew toward both sides of cerebral falx, 21 cases had this type.

Thirty-four cases underwent a DSA examination, and 17 cases were discovered to have significant tumor stain in the venous phase. Seventeen of these had a double blood supply through internal and external carotid artery system, 13 cases had blood supply through the external carotid artery, in whom tumor staining was evidently reduced after undergoing arterial embolization, one case's blood supply was only from an ipsilateral ophthalmic artery from the internal carotid artery system. We did not perform resonance studies for the venous phase.

\section{Surgical methods}

Preoperative intervention

For the 21 cases who were shown (by MRI) to have significant peritumoral edema, $20 \mathrm{mg}$ of dexamethasone and $250 \mathrm{~mL}$ of $20 \%$ mannitol, Q12h-Q8h was administered daily for 3 days prior to surgery.

\section{Anesthesia and position}

All surgeries were performed with endotracheal intubation under general anesthesia. The 42 cases whose tumors were located at the anterior third of cerebral falx were in a horizontal position, their heads were slightly higher than the horizontal line of the body by $15^{\circ}$; the 55 cases whose tumors were located at the middle third or over both anterior and middle thirds of cerebral falx had their heads lifted up and their necks anteflexed, allowing the head and neck to be angled to the horizontal plane by $126^{\circ}-40^{\circ}$; the 17 cases whose tumors were located at the posterior third of cerebral falx were in a prone position. The heads of the 71 type-I cases were slightly inclined to the opposite side of tumor by $5^{\circ}-10^{\circ}$, allowing the surgical field to be at the highest position, which facilitates the venous return. The heads of the 34 type-II cases were inclined to the opposite side of tumor by $10^{\circ}-20^{\circ}$. The heads of the 21 type-III cases were inclined to the side where the tumor was smaller, surgery 
was first performed at the side where the tumor was bigger. An adjustment could be made according to the specific location of the tumor during surgery.

\section{Surgical incision}

The incision and approach were selected based on the location of the tumor, as shown by MRI, the incision was overmidline and horseshoe-shaped, and its size exceeded each border of the tumor by $2-3 \mathrm{~cm}$.

\section{Microsurgery}

The principle was that the approach through longitudinal fissure was chosen according to the distribution of venous return vessels shown by DSA, in order to avoid damaging the bridging vein and brain functional region. The details were as follows: 1) for those whose tumors were at the anterior or middle third of cerebral falx, the central sulcus vein was avoided when approaching through longitudinal fissure; 2) the basilar part of the tumor was fulgurated along the cerebral falx to block the blood supply; for those whose tumors were large, the tumor was first resected inside the envelope, then the periphery was resected once its volume had been reduced; 3) a cotton sponge was placed on the separation surface in order to prevent the peripheral cerebral cortex from being injured and to protect the pericallosal artery beneath. The operative blood loss of 126 cases ranged from $190 \mathrm{~mL}$ to $905 \mathrm{~mL}$, averaging $385 \mathrm{~mL}$. We did not use the ultrasonic vacuum during the wound resection.

\section{Results \\ Extent of resection of tumors}

Extent of resection of tumors was in accordance with Simpson criterion ${ }^{3}$ : G1 (thoroughly resected) 13 cases; G2 (entirely resected) 105 cases; G3 (entirely resected to naked eye) four cases (the tumor tightly adhered to the central sulcus vein, incompletely separated); G4 (sub-totally resected or partly resected) four cases (multiple tumors, the smaller part of tumor at the frontal lobe was not treated in the first phase); and there were no G5 cases (Table 1).

\section{Pathological results}

There were fifty-eight cases of endothelial-type (46.1\%), 29 cases of fibro-type (23.0\%), 14 cases of blood vessel-type (11.1\%), 13 cases of sand grain-type (10.3\%), nine cases of mixed type (7.1\%), and three cases of atypical meningioma (2.4\%). All of the 126 cases underwent immunohistochemical analysis and the results were as follows: $\operatorname{EMV}(+)$ : 88 cases, Vimentin(+): 84 cases, S-100(+): 59 cases,
Table I The extent of resection of the parafalcine meningiomas

\begin{tabular}{|c|c|c|}
\hline $\begin{array}{l}\text { Resection } \\
\text { grade }\end{array}$ & Simpson criterion & $\begin{array}{l}\text { Case } \\
\text { number }\end{array}$ \\
\hline I & $\begin{array}{l}\text { Macroscopically complete removal of tumor, } \\
\text { with excision of its dural attachment, and } \\
\text { of any abnormal bone. Includes resection of } \\
\text { venous sinus if involved. }\end{array}$ & 13 \\
\hline II & $\begin{array}{l}\text { Macroscopically complete removal of tumor } \\
\text { and its visible extensions with coagulation of its } \\
\text { dural attachment. }\end{array}$ & 105 \\
\hline III & $\begin{array}{l}\text { Macroscopically complete removal of the } \\
\text { intradural tumor, without resection or } \\
\text { coagulation of its dural attachment or its } \\
\text { extradural extensions. }\end{array}$ & 4 \\
\hline IV & Partial removal, leaving intradural tumor in situ. & 4 \\
\hline V & Simple decompression, with or without biopsy. & 0 \\
\hline
\end{tabular}

CD34(+): 48 cases, P53(+): 19 cases, HMB 45(+): nine cases, and CD68(+): five cases. Ki-67 index was between $<1 \%$ and $12 \%$, averaging $2.4 \%$.

\section{Complications}

Eighteen patients developed hypokinesia of the contralateral limbs after surgery, which resolved after treatment. Although the patients were administered sodium valproate for conventional prevention of seizures after surgery, 12 patients still had an epileptic seizure, which resolved after medication. Five cases developed a postoperative secondary tumor cavityhematoma, which was resolved by hematoma clearance. Four cases developed acute pancreatitis on day 2 after surgery, which was resolved by conservative therapy.

\section{Discussion}

There are often no typical symptoms in the early stage of parafalcine meningiomas, and the tumor size is usually quite large by the time it is diagnosed. Maximizing the removal of tumors and reducing the incidence of surgical complications and tumor recurrence rates have always been the goal for neurosurgeons. With the advancement of modern examination techniques and the development of neurosurgical microscopy, the safety and effectiveness of surgical treatments of parafalcine meningiomas have been significantly improved. In recent years, articles on analyses of meningioma surgical techniques have been increasing year by year (Figure 1). The origins of these studies are mainly concentrated in East Asia, Europe, and North America (Figure 2).

\section{Design of skin flap and bone flap}

Out of 126 meningioma cases, the 21 cases with posterior third type were placed in a prone position and surgery was 


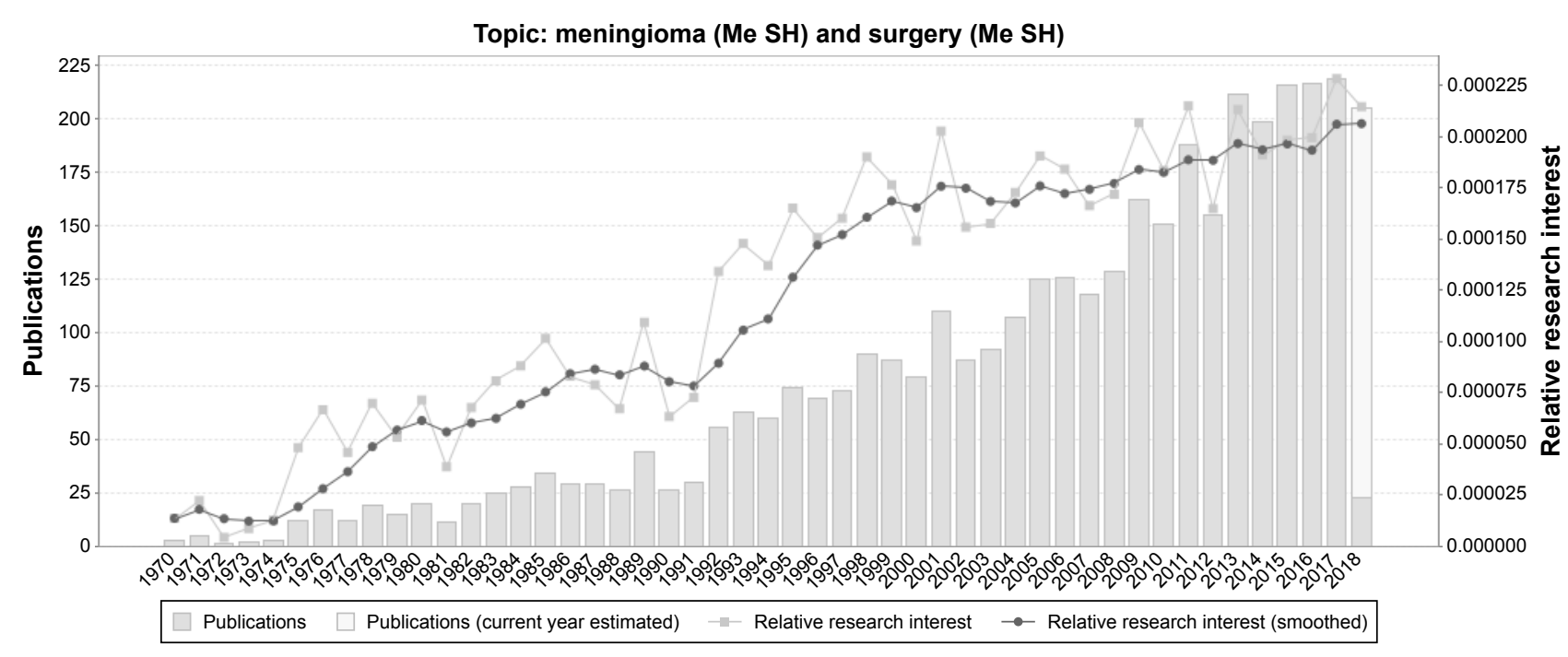

Figure I A timeline of the publications related to meningioma and surgery (the date of the end-point is March 2018). Me SH, Medical Subject Headings.

performed using a horseshoe-shaped incision striding over the midline; the remaining anterior and middle third type cases underwent surgery using a horseshoe-shaped incision inside the hairline. Skin flap: the size of skin flap was determined based on the depth of tumor, generally, the skin flap should exceed each border of tumor by $2 \mathrm{~cm}$. The skin flap should be bigger if the tumor is close to the cingulate gyrus in order to avoid traction injury of bridging vein and central sulcus vein when revealing the tumor. Bone flap: its size should exceed the border of tumor by $2 \mathrm{~cm}$, and its medial border should exceed the lateral border of the sagittal sinus, the position of the bridging vein in the surgical field should be determined first when the dura mater is opened and compared with the preoperative DSA in order to determine the surgical approach. The position, shape, and distribution of the central sulcus vein are determined based on the results of MRI and DSA. ${ }^{4}$

\section{Tumor resection}

The sagittal sinus should be well-protected when the tumor is revealed to prevent it from rupturing. A small rupture of the sagittal sinus should be repaired by using hemostatic gauze and adhesion of gelatin sponge plus biogel; as for a larger rupture, it should be fixed by suspended compression. When the tumor is small, as was the case in 34 patients in this group whose tumors' maximum diameter was less than $2 \mathrm{~cm}$,

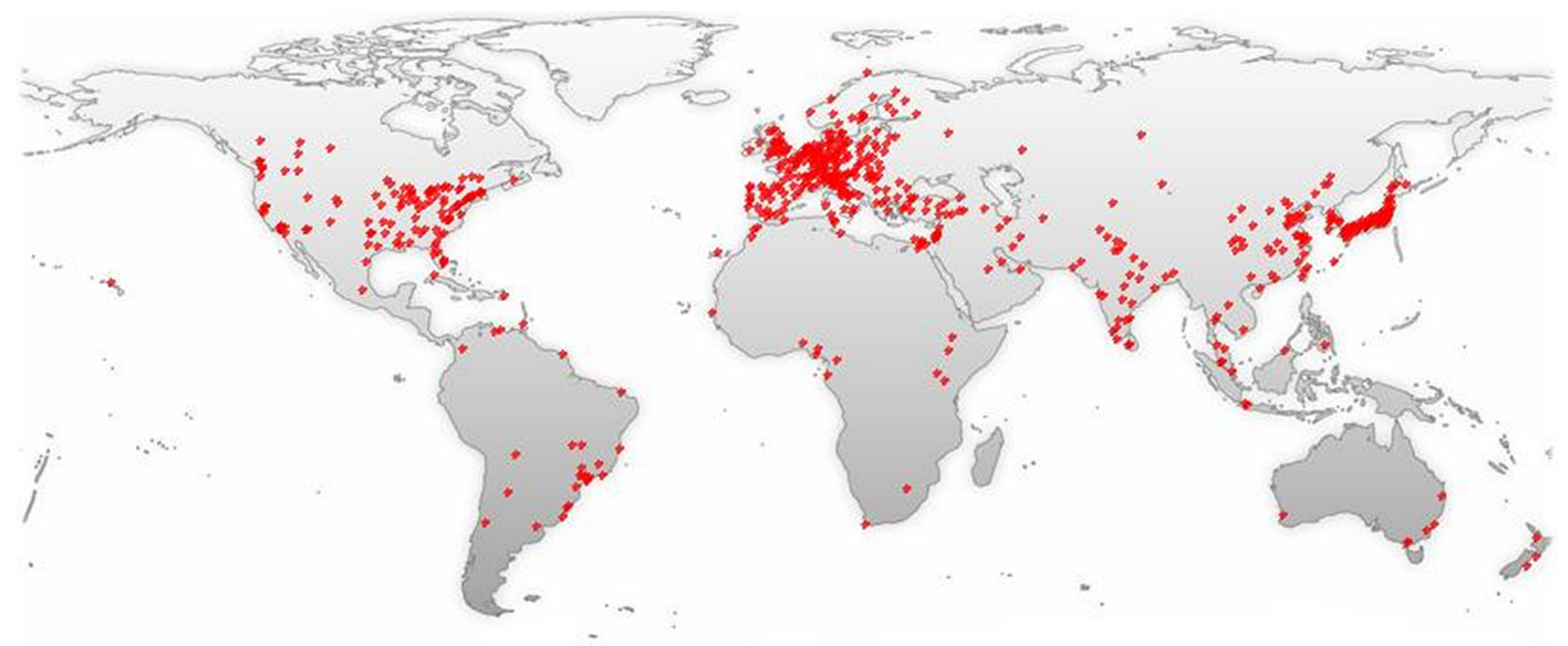

Figure 2 A world map with the global distribution of publications related to the surgical treatments of meningiomas based on the analysis of their geolocational data. Note: "Meningioma/surgery"[Mesh] has been indexed in the PubMed database and 6232 abstracts identified and semantically analyzed with GoPubMed. 
the part of the tumor that is adhered to the cerebral falx should be separated and its basilar part should be fulgurated first, then the peritumoral part should be separated; meanwhile the pericallosal artery, possibly located at the deep layer of the tumor, should be carefully protected. In the case of a large tumor and wide basilar part, the envelope should be cut open first if the blood supply is not rich, then the tumor should be emptied by tumor-fetching pincer and ultrasound knife, its basilar part should be fulgurated, followed by a piecemeal resection of collapsed cyst wall once its volume had been reduced.

Out of the 13 cases whose tumors' maximum diameter was greater than $4 \mathrm{~cm}$, eight cases had an extremely rich blood supply, as a result, the adhesion was separated to block the blood supply first, then a piecemeal resection was performed inside the cyst, with a satisfactory effect. The fulguration of tumor base, intra-envelope resection of tumor, and other procedures were conducted alternatively, while avoiding damage to the peripheral brain tissues, as much as possible. Oyama et al believe that the postoperative recurrence rate could be reduced by resection of meningioma with a wide base along with cerebral falx or by fulguration of the adhered part. ${ }^{2}$ As for the type-II and III meningioma, the side where the tumor focus is bigger should be resected first, then the part adhered to the cerebral falx should be cut open, the contralateral part can be resected through the coloboma hole of cerebral falx due to resection. ${ }^{5}$ If there are large tumors at both sides, Yu et al believe that the cerebral dura mater should be cut open along both sides and flipped toward the midline, then resected respectively, followed by a circular resection of the cerebral falx, finally. ${ }^{6}$ There were no cases of meningiomas of identical size at both sides in our study. Of note, during falcine meningioma surgery, attention should be paid to cardiac monitoring due to the risk that handling of the falx could provoke cardiac arrest. As for our study, there were no cases characterized by this occurrence.

\section{Handling of feeding artery}

Parafalcine meningiomas receive blood supply from both the internal and external carotid artery; with an abundant blood supply, the key to successful surgery is to reduce hemorrhage.? We believe that: 1) preoperative DSA should be performed to determine the origin of arterial supply and situation of blood supply of tumor, and compression of pericallosal artery should be performed, simultaneously, the supplying artery should be embolized to facilitate the reduction of intraoperative hemorrhage and improve surgical safety and thorough removal, the surgery may be performed within 3-7 days after embolization; ${ }^{8} 2$ ) it is crucial to ensure the surgical field is kept at the highest position to facilitate the venous return and reduction of intracranial pressure; 3 ) before the dura mater is cut open, a fast intravenous drip of $250 \mathrm{~mL}$ of $20 \%$ mannitol should be administered, if the intracranial pressure has not improved significantly, proper hyperventilation should be used; 4) the cerebral dura mater should be separated carefully by stripper when the bone flap is turned over, the rupture of dura mater should be avoided; the sagittal sinus margin should be approached adequately while the sagittal sinus should be protected in order to avoid hemorrhage when the cerebral dura mater is being cut open near the midline; 5) as for the supplying vessel of the tumor, the principle that the artery should be treated first and then the vein should be blocked, should be followed according to the arterial distribution shown by DSA, otherwise, the patient is at a risk of fast congestion and expansion of tumor and then massive hemorrhage. One hundred and twenty-six patients with parafalcine meningiomas in this group were treated with the previously mentioned measures, with an average blood loss of only $410 \mathrm{~mL}$. Of note, the role of preoperative embolization in meningioma management remains controversial. It has been shown to offer benefits such as decreased blood loss and "softening of the tumor" during subsequent resection. However, the actual benefits remain unclear. Further randomized trials with large sample sizes are needed.

\section{Protection of central sulcus vein}

If the central sulcus vein is injured, the patient usually develops severe contralateral limb paralysis after surgery.

Among the 126 cases, there were no central sulcus vein injuries. There were four patients whose tumor tissue was tightly adhered to the central sulcus vein and very difficult to separate, consequently, the tumor tissues around the vein were resected in a piecemeal manner and properly fulgurated, finally, a thin slice of tumor was left, normal saline was injected continuously during the surgery to reduce the temperature, and the cotton slice was used for protection.

The central sulcus vein may be injured and bleeding due to collapse and separation after tumor removal, therefore, electrocoagulation should not be conducted at that time, a gelatin sponge pad should be used for compression, in order to prevent thromboembolism, the traction of cerebral tissues should be avoided as much as possible when the tumor is revealed, so as to protect the bridging vein's drainage toward the sagittal sinus; the surgical approach is evidently blocked a proper fulguration should be done to the bridging vein in the non-functional region. 


\section{Treatment of affected sagittal sinus}

The treatment of affected sagittal sinus is a difficult point in surgery, and there are many controversies regarding this. The authors' experience in this regard follows: 1) for the tumors that only invade the sagittal sinus wall and not the sagittal sinus, after resection of the whole tumor, we would use a low-power electric coagulation knife to fulgurate the sagittal antral wall and cerebral falx that adhered to the tumor several times, which can prevent tumor recurrence for a long time. It should be noted that when the sagittal sinus is repeatedly fulgurated, cold normal saline should be used to reduce the temperature, so as to avoid possible sagittal sinus thrombosis. 2) For tumors that invade the sagittal sinus and even those that result in the sagittal sinus being partially occluded, if it is located in the anterior third, we would ligate and resect the affected sagittal sinus and cerebral falx, which will not cause adverse consequences; if the tumor is located in the middle or anterior third of the sagittal sinus, we would totally resect the tumor, then use a low-power electric coagulation knife to fulgurate the sagittal antral wall adhered to the tumor repeatedly, and perform radiotherapy or gamma knife treatment postoperatively. If the tumor then gradually increases and slowly occludes the sagittal sinus, and good collateral circulation has been established, we would perform phase II surgery in order to completely remove the tumor. 3) For patients with complete sagittal sinus occlusion, after confirming that collateral circulation is satisfactory, we would excise the segment without considering reconstruction, regardless of which resection is occluded.

\section{Possible causes of recurrence and its treatment}

With parafalcine meningiomas, the tumor can grow back if the residue of it is left at the primary site after surgery. One reason is that the tumor is deeply buried under the cerebral hemisphere cortex, which does not permit an adequately exposed surgical field, so it is very difficult to achieve a radical resection, which in turn leads to recurrence of the tumor from the base; the other reason is that the meningioma itself is characterized by invasiveness and polycentric growth and other biological characteristics, particularly for the anaplastic, atypical, and malignant meningioma, some oncocytes so there may be some tumor residual near the primary focus. ${ }^{9}$ The first choice of treatment for recurrence of meningioma is still surgery, postoperative therapy could be aided by radiotherapy, eg, gamma knife and X knife. ${ }^{10}$

\section{Acknowledgments}

This study was supported by the grants from Beijing Municipal Science and Technology Project (No. Z151100003915109), Capital Public Health Education, Beijing Science and Technology Program (No. Z171100000417028), and Chinese Academy of Medical Sciences Initiative for Innovative Medicine (No 2017-I2M-3-020). The funders had no role in study design, data collection and analysis, decision to publish, or preparation of the manuscript.

\section{Disclosure}

As this is just a retrospective study that does not involve any interventions, ethical approval was not necessary. The authors report no conflicts of interest in this work.

\section{References}

1. Bi WL, Zhang M, Wu WW, Mei Y, Dunn IF. Diagnostic Meningioma Genomics: Prognostic, and Therapeutic Applications. Front Surg. 2016;3:40.

2. Oyama H, Kito A, Maki H, Hattori K, Noda T, Wada K. Surgical results of parasagittal and falx meningioma. Nagoya JMed Sci. 2012;74(1-2): 211-216.

3. Oya S, Kawai K, Nakatomi H, Saito N. Significance of Simpson grading system in modern meningioma surgery: integration of the grade with MIB-1 labeling index as a key to predict the recurrence of WHO Grade I meningiomas. J Neurosurg. 2012;117(1):121-128.

4. Huang SQ, Liang BL, Xie BK, Yuan JP, Zhong JL, Ye RX. MRI performance and diagnosis of meningioma - a report of 126 cases. Ai Zheng. 2004;23(11):1329-1333.

5. Ausman JI. Strategy and technique for removal of a falcine meningioma in a patient presenting with bilateral meningiomas. Surg Neurol. 2001;55(4):204-208.

6. L1 Y. Clinical Research of Microsurgical Removal of Falx Meningiomas. Chinese Journal of General Practice. 2013;11:1387-1389.

7. Maiuri F, Donzelli R, de Divitiis O, Fusco M, Briganti F. Anomalous meningeal branches of the ophthalmic artery feeding meningiomas of the brain convexity. Surg Radiol Anat. 1998;20(4):279-284.

8. Uetani H, Akter M, Hirai T, et al. Can $3 \mathrm{~T}$ MR angiography replace DSA for the identification of arteries feeding intracranial meningiomas? AJNR Am J Neuroradiol. 2013;34(4):765-772.

9. Strassner C, Buhl R, Mehdorn HM. Recurrence of intracranial meningiomas: did better methods of diagnosis and surgical treatment change the outcome in the last 30 years? Neurol Res. 2009;31(5):478-482.

10. Sheehan JP, Lee CC, Xu Z, Przybylowski CJ, Melmer PD, Schlesinger D. Edema following Gamma Knife radiosurgery for parasagittal and parafalcine meningiomas. J Neurosurg. 2015;123(5):1287-1293. 
OncoTargets and Therapy

\section{Publish your work in this journal}

OncoTargets and Therapy is an international, peer-reviewed, open access journal focusing on the pathological basis of all cancers, potential targets for therapy and treatment protocols employed to improve the management of cancer patients. The journal also focuses on the impact of management programs and new therapeutic agents and protocols on

perspectives such as quality of life, adherence and satisfaction. The manuscript management system is completely online and includes a very quick and fair peer-review system, which is all easy to use. Visit http://www.dovepress.com/testimonials.php to read real quotes from published authors.

Submit your manuscript here: http://www.dovepress.com/oncotargets-and-therapy-journal 